Geometry 85 Topology

Volume 6 (2002) 69-89

Published: 1 March 2002

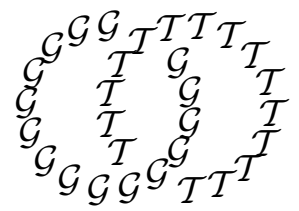

\title{
Bounded cohomology of subgroups of mapping class groups
}

\author{
Mladen Bestuina \\ KOJI FuJIWARA \\ Mathematics Department, University of Utah \\ 155 South 1400 East, JWB 233 \\ Salt Lake City, UT 84112, USA \\ and \\ Mathematics Institute, Tohoku University \\ Sendai, 980-8578, Japan
}

Email: bestvina@math.utah.edu and fujiwara@math.tohoku.ac.jp

\begin{abstract}
We show that every subgroup of the mapping class group $M C G(S)$ of a compact surface $S$ is either virtually abelian or it has infinite dimensional second bounded cohomology. As an application, we give another proof of the FarbKaimanovich-Masur rigidity theorem that states that $M C G(S)$ does not contain a higher rank lattice as a subgroup.
\end{abstract}

AMS Classification numbers Primary: 57M07, 57N05

Secondary: 57M99

Keywords: Bounded cohomology, mapping class groups, hyperbolic groups

Proposed: Joan Birman

Seconded: Dieter Kotschick, Steven Ferry

(C) Geometry $\& \mathcal{G}$ Topology $\mathcal{P}$ ublications
Received: 15 December 2000

Revised: 28 February 2002 


\section{Introduction}

When $G$ is a discrete group, a quasi-homomorphism on $G$ is a function $h: G \rightarrow$ $\mathbb{R}$ such that

$$
\Delta(h):=\sup _{\gamma_{1}, \gamma_{2} \in G}\left|h\left(\gamma_{1} \gamma_{2}\right)-h\left(\gamma_{1}\right)-h\left(\gamma_{2}\right)\right|<\infty .
$$

The number $\Delta(h)$ is the defect of $h$. Let $\mathcal{V}(G)$ be the vector space of all quasihomomorphisms $G \rightarrow \mathbb{R}$. By $B D D(G)$ and respectively $H O M(G)=H^{1}(G ; \mathbb{R})$ denote the subspaces of $\mathcal{V}(G)$ consisting of bounded functions and respectively homomorphisms. Note that $B D D(G) \cap \operatorname{HOM}(G)=0$. We will be concerned with the quotient spaces

$$
\begin{gathered}
Q H(G)=\mathcal{V}(G) / B D D(G) \quad \text { and } \\
\widetilde{Q H}(G)=\mathcal{V}(G) /(B D D(G)+H O M(G)) \cong Q H(G) / H^{1}(G ; \mathbb{R}) .
\end{gathered}
$$

There is an exact sequence

$$
0 \rightarrow H^{1}(G ; \mathbb{R}) \rightarrow Q H(G) \rightarrow H_{b}^{2}(G ; \mathbb{R}) \rightarrow H^{2}(G ; \mathbb{R})
$$

where $H_{b}^{2}(G ; \mathbb{R})$ denotes the second bounded cohomology of $G$ (for the background on bounded cohomology the reader is referred to [14] and [24]). Since $\widetilde{Q H}(G)$ is the quotient $Q H(G) / H^{1}(G ; \mathbb{R})$ we see that $\widetilde{Q H}(G)$ can be also identified with the kernel of $H_{b}^{2}(G ; \mathbb{R}) \rightarrow H^{2}(G ; \mathbb{R})$. If $G \rightarrow G^{\prime}$ is an epimorphism then the induced maps $Q H\left(G^{\prime}\right) \rightarrow Q H(G)$ and $\widetilde{Q H}\left(G^{\prime}\right) \rightarrow \widetilde{Q H}(G)$ are injective.

Calculations of $\widetilde{Q H}(G)$ have been made for many groups $G$. In all such cases $\widetilde{Q H}(G)$ is either 0 or infinite dimensional. $\widetilde{Q H}(G)$ vanishes when $G$ is amenable (see [14]) and also notably when $G$ is a cocompact irreducible lattice in a semisimple Lie group of real rank $>1$ [5].

In the sequence of papers $[8,11,12]$ the second author has established a method for showing that $\widetilde{Q H}(G)$ is infinite dimensional for groups $G$ acting on hyperbolic spaces and satisfying certain additional conditions. This represents a generalization of the argument of Brooks [2] that $\operatorname{dim} \widetilde{Q H}(G)=\infty$ when $G$ is a nonabelian free group. Theorem 1 can be viewed as a refinement of that method.

Not every group $G$ acting on a hyperbolic space has $\operatorname{dim} \widetilde{Q H}(G)=\infty$. A nontrivial example is provided by an irreducible cocompact lattice in $S L_{2}(\mathbb{R}) \times$ $S L_{2}(\mathbb{R})$ that acts (discretely) on the product $\mathbb{H}^{2} \times \mathbb{H}^{2}$ of two hyperbolic planes. 
Notice that the action given by projecting to a single factor is highly nondiscrete. Our contribution in this paper is to identify what we believe to be the "right" condition on the action that guarantees $\operatorname{dim} \widetilde{Q H}(G)=\infty$. The condition is termed WPD ("weak proper discontinuity").

The main application is to the action of mapping class groups on curve complexes. These were shown to be hyperbolic by Masur-Minsky [22]. The action is far from discrete - indeed, the vertex stabilizers are infinite. However, we will show that $W P D$ holds for this action. As a consequence we will deduce the rigidity theorem of Farb-Kaimanovich-Masur that mapping class groups don't contain higher rank lattices as subgroups. More generally, if $\Gamma$ is not virtually abelian and $\operatorname{dim} \widetilde{Q H}(\Gamma)<\infty$ then $\Gamma$ does not occur as a subgroup of a mapping class group. In particular, if the mapping class group $M C G(S)$ is not virtually abelian, then $\operatorname{dim} \widetilde{Q H}(G)=\infty$. This settles Morita's Conjectures 6.19 and $6.21[25]$ in the affirmative.

We now proceed with a review of hyperbolic spaces and we introduce some terminology needed in the paper.

When $X$ is a connected graph, we consider the path metric $d=d_{X}$ on $X$ by declaring that each edge has length 1 . A geodesic arc is a path whose length is equal to the distance between its endpoints. A bi-infinite geodesic is a line in $X$ such that every finite segment is geodesic. Recall [13] that $X$ is said to be $\delta$-hyperbolic if for any three geodesic $\operatorname{arcs} \alpha, \beta, \gamma$ in $X$ that form a triangle we have that $\alpha$ is contained in the $\delta$-neighborhood of $\beta \cup \gamma$.

A map $\phi: Y \rightarrow X$ from a metric space $Y$ is a $(K, L)$-quasi-isometric (qi) embedding if

$$
\frac{1}{K} d_{Y}\left(y, y^{\prime}\right)-L \leq d_{X}\left(\phi(y), \phi\left(y^{\prime}\right)\right) \leq K d_{Y}\left(y, y^{\prime}\right)+L
$$

for all $y, y^{\prime} \in Y$. A $(K, L)$-quasi-geodesic (or just quasi-geodesic when $(K, L)$ are understood) is a $(K, L)$-qi embedding of an interval (finite or infinite). A fundamental property of $\delta$-hyperbolic spaces is that there is $B=B(K, L, \delta)$ such that any two finite $(K, L)$-quasi-geodesics with common endpoints are within $B$ of each other, and also any two bi-infinite quasi-geodesics that are finite distance from each other are within $B$ of each other. A qi embedding $Y \rightarrow X$ is a quasi-isometry if the distance between points of $X$ and the image of the map is uniformly bounded.

An isometry $g$ of $X$ is axial if there is a bi-infinite geodesic (called an axis of $g$ ) on which $g$ acts as a nontrivial translation. Any axis of $g$ is contained in the $2 \delta$-neighborhood of any other axis of $g$. More generally, an isometry $g$ of 
$X$ is hyperbolic if it admits an invariant quasi-geodesic (we will refer to it as a quasi-axis or a $(K, L)$-quasi-axis if we want to emphasize $K$ and $L)$. We will often blur the distinction between a quasi-axis and its image. There are easy examples of hyperbolic isometries that are not axial, but whose squares are axial (eg, take the "infinite ladder" consisting of two parallel lines and rungs joining corresponding integer points, and the isometry that interchanges the lines and moves rungs one unit up). When the graph is allowed to be locally infinite, there are similar examples of hyperbolic isometries none of whose powers are axial. In our main application, the action of the mapping class group on the curve complex, it is unknown whether powers of hyperbolic elements are axial. We are thankful to Howie Masur and Yair Minsky for bringing up this point. Note that any two $(K, L)$-quasi-axes of $g$ are within $B(K, L, \delta)$ of each other.

Every quasi-axis of $g$ is oriented by the requirement that $g$ acts as a positive translation. We call this orientation the g-orientation of the quasi-axis. Of course, the $g^{-1}$-orientation is the opposite of the $g$-orientation. More generally, any sufficiently long $(K, L)$-quasi-geodesic arc $J$ inside the $B(K, L, \delta)-$ neighborhood of a $(K, L)$-quasi-axis $\ell$ of $g$ has a natural orientation given by $g$ : a point of $\ell$ within $B(K, L, \delta)$ of the terminal endpoint of $J$ is ahead (with respect to the $g$-orientation of $\ell$ ) of a point of $\ell$ within $B(K, L, \delta)$ of the initial endpoint of $J$. We call this orientation of $J$ the $g$-orientation. We say that two quasi-geodesic arcs are $C$-close if each is contained in the $C$-neighborhood of the other, and we say that two oriented quasi-geodesic arcs are oriented $C-$ close if they are $C$-close and the distance between their initial and also their terminal endpoints is $\leq C$.

Definition When $g_{1}$ and $g_{2}$ are hyperbolic elements of $G$ we will write

$$
g_{1} \sim g_{2}
$$

if for an arbitrarily long segment $J$ in a $(K, L)$-quasi-axis for $g_{1}$ there is $g \in G$ such that $g(J)$ is within $B(K, L, \delta)$ of a $(K, L)$-quasi-axis of $g_{2}$ and $g: J \rightarrow$ $g(J)$ is orientation-preserving with respect to the $g_{1}$-orientation on $J$ and the $g_{2}$-orientation on $g(J)$.

Replacing the constant $B(K, L, \delta)$ by a larger constant would not change the concept since for every $C>0$ there is $C^{\prime}>0$ such that for any $(K, L)$-quasigeodesic arc $J$ contained in the $C$-neighborhood of a $(K, L)$-quasi-geodesic $\ell$ it follows that the arc obtained by removing the $C^{\prime}$-neighborhood of each vertex is contained in the $B(K, L, \delta)$-neighborhood of $\ell$. Similarly, the concept does not depend on the choice of $(K, L)$. In particular: 
- $\sim$ is an equivalence relation.

- $g_{1} \sim g_{2}$ if and only if $g_{1}^{k} \sim g_{2}^{l}$ for any $k, l$ with $k l>0$.

- If $g_{1}$ and $g_{2}$ have positive powers which are conjugate in $G$ then $g_{1} \sim g_{2}$.

Under our condition WPD (see Section 3) the converse of the third bullet also holds.

Definition We say that the action of $G$ on $X$ is nonelementary if there exist at least two hyperbolic elements whose $(K, L)$-quasi-axes do not contain rays within finite distance of each other (this distance can be taken to be $B(K, L, \delta)$ ). The two hyperbolic elements are then called independent.

Theorem 1 Suppose a group $G$ acts on a $\delta$-hyperbolic graph $X$ by isometries. Suppose also that the action is nonelementary and that there exist independent hyperbolic elements $g_{1}, g_{2} \in G$ such that $g_{1} \nsim g_{2}$.

Then $\widetilde{Q H}(G)$ is infinite dimensional.

Remark Special cases of this theorem are discussed in the earlier papers of the second author:

- [8] $G$ is a word-hyperbolic group acting on its Cayley graph,

- [11] the action of $G$ on $X$ is properly discontinuous,

- [12] $G$ is a graph of groups acting on the associated Bass-Serre tree.

Proposition 2 Under the hypotheses of Theorem 1 there is a sequence $f_{1}, f_{2}, \cdots \in G$ of hyperbolic elements such that

- $f_{i} \nsim f_{i}^{-1}$ for $i=1,2, \cdots$, and

- $f_{i} \nsim f_{j}^{ \pm 1}$ for $j<i$.

Proof Since $g_{1}$ and $g_{2}$ are independent, we may replace $g_{1}, g_{2}$ by high positive powers of conjugates to ensure that the subgroup $F$ of $G$ generated by $g_{1}, g_{2}$ is free with basis $\left\{g_{1}, g_{2}\right\}$, each nontrivial element of $F$ is hyperbolic, and $F$ is quasi-convex with respect to the action on $X$ (see [13, Section 5.3]). We will call such free subgroups Schottky groups. Let $T$ be the Cayley graph of $F$ with respect to the generating set $\left\{g_{1}, g_{2}\right\}$. Then $T$ is a tree and each oriented edge has a label $g_{i}^{ \pm 1}$. Choose a basepoint $x_{0} \in X$ and construct an $F$-equivariant map $\Phi: T \rightarrow X$ that sends 1 to $x_{0}$ and sends each edge to a geodesic arc. Quasi-convexity implies that $\Phi$ is a $(K, L)$-quasi-isometric embedding for some 
$(K, L)$ and in particular for every $1 \neq f \in F$ the $\Phi$-image of the axis of $f$ in $T$ is a $(K, L)$-quasi-axis of $f$. By $\ell_{i}$ denote the axis of $g_{i}$ in $T, i=1,2$.

Choose positive constants

$$
0 \ll n_{1} \ll m_{1} \ll k_{1} \ll l_{1} \ll n_{2} \ll m_{2} \ll \cdots
$$

and define

$$
f_{i}=g_{1}^{n_{i}} g_{2}^{m_{i}} g_{1}^{k_{i}} g_{2}^{-l_{i}}
$$

for $i=1,2,3, \cdots$.

Claim $1 f_{1} \nsim f_{2}$.

The key to the proof is the following observation. If $K^{\prime}, L^{\prime}, C$ are fixed and the exponents $n_{1}, m_{1}, \cdots, k_{2}, l_{2}$ are chosen suitably large, then for any sufficiently long $f_{2}$-oriented segment $S$ in the axis $\ell_{2} \subset T$ of $f_{2}$ and any orientation preserving $\left(K^{\prime}, L^{\prime}\right)$-qi embedding $\phi: S \rightarrow \ell_{1}$ (with respect to the $f_{1}$-orientation of $\ell_{1}$ ) there is a subsegment $S^{\prime}$ of $S$ containing a string of $\geq C$ edges labeled $g_{2}$ whose image (pulled tight) is a segment in $\ell_{1}$ consisting only of edges labeled $g_{1}$ (this is true because $m_{2} \gg n_{1}+m_{1}+k_{1}+l_{1}$ so the image will contain a whole fundamental domain for the action of $f_{1}$ on $\ell_{1}$ ). Figure 1 illustrates the situation.

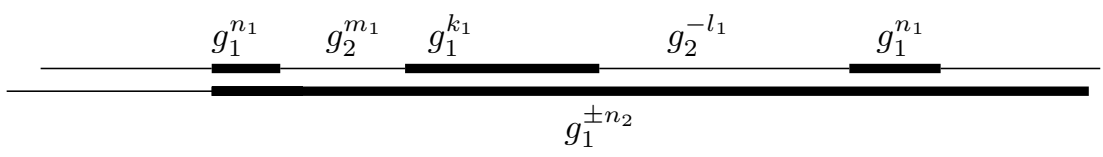

Figure 1: Thick (thin) lines represent strings of edges labeled $g_{1}\left(g_{2}\right)$.

Now assuming that $f_{1} \sim f_{2}$ let $I_{2} \subset \ell_{2}$ be a long arc, let $J=\Phi\left(I_{2}\right)$, and let $g \in G$ be such that $g(J)$ is $B(K, L, \delta)$-close to the $(K, L)$-quasi-axis $f_{1}\left(\ell_{1}\right)$ of $f_{1}$, with matching orientations. Choose an arc $I_{1} \subset \ell_{1}$ so that $\Phi\left(I_{1}\right)$ is $B(K, L, \delta)$-close to $g(J)$. Then there is a $\left(K^{\prime}, L^{\prime}\right)$-quasi-isometry $I_{2} \rightarrow I_{1}$ obtained by composing $I_{2} \rightarrow \Phi\left(I_{2}\right)=J \rightarrow g(J) \rightarrow \Phi\left(I_{1}\right) \rightarrow I_{1}$ and $\left(K^{\prime}, L^{\prime}\right)$ does not depend on the choices of $n_{1}, m_{1}, \cdots, k_{2}, l_{2}$, only on $\delta, T$, and $\Phi$. Combining this with the observation above, we conclude that $g$ takes a long segment in an axis of a conjugate of $g_{2}$ uniformly close to an axis of a conjugate of $g_{1}$ with matching orientation, contradicting the assumption that $g_{1} \nsim g_{2}$.

Similarly, $f_{i} \not f_{j}$ for $i \neq j$.

Claim $2 f_{1} \nsim f_{2}^{-1}$. 
The proof is similar to the proof of Claim 1, only now one uses $l_{2} \gg n_{1}+m_{1}+$ $k_{1}+l_{1}$.

Similarly, $f_{i} \nsim f_{j}^{-1}$ for $i \neq j$.

Claim 3 If in addition $g_{1} \nsim g_{2}^{-1}$ then $f_{1} \nsim f_{1}^{-1}$.

If $f_{1} \sim f_{1}^{-1}$, we obtain the situation pictured in Figure 2 where a long string of $g_{1}$ 's is close to a long string of $g_{2}$ 's with either the same or opposite orientation. Note that it is possible that all such pairs of strings have opposite orientation so the assumption $g_{1} \nsim g_{2}^{-1}$ is necessary.

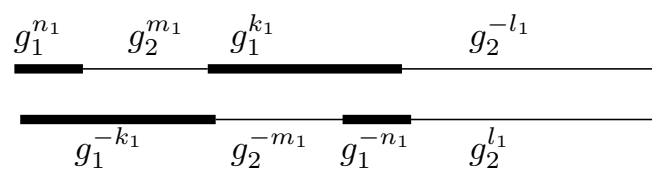

Figure 2: Whenever thick and thin lines are close, they are anti-parallel.

Similarly, if $g_{1} \nsim g_{2}^{-1}$ then $f_{i} \nsim f_{i}^{-1}$ for all $i$.

We now finish the proof. If $g_{1} \nsim g_{2}^{-1}$ then the above claims conclude the argument. Otherwise, note that by Claims 1 and 2 we have $f_{1} \nsim f_{2}^{ \pm 1}$. Now replace $\left(g_{1}, g_{2}\right)$ by $\left(f_{1}, f_{2}\right)$ and repeat the construction.

\section{Proof of Theorem 1}

We will only give a sketch of the proof since it is a minor generalization of results in $[8,11,12]$ and the proof uses the same techniques.

We start by recalling the basic construction of quasi-homomorphisms in this setting. The model case of the free group is due to Brooks [2].

Let $w$ be a finite (oriented) path in $X$. By $|w|$ denote the length of $w$. For $g \in G$ the composition $g \circ w$ is a copy of $w$. Obviously $|g \circ w|=|w|$.

Let $\alpha$ be a finite path. We define

$$
|\alpha|_{w}=\{\text { the maximal number of non-overlapping copies of } w \text { in } \alpha\} .
$$

Suppose that $x, y \in X$ are two vertices and that $W$ is an integer with $0<$ $W<|w|$. We define the integer

$$
c_{w, W}(x, y)=d(x, y)-\inf _{\alpha}\left(|\alpha|-W|\alpha|_{w}\right),
$$


where $\alpha$ ranges over all paths from $x$ to $y$. Note that if $\alpha$ is such a path that contains a subpath whose length is large compared to the distance between its endpoints, then replacing this subpath by a geodesic arc between the endpoints produces a new path with smaller $|\alpha|-W|\alpha|_{w}$. This observation leads to the following lemma.

Lemma 3 [11, Lemma 3.3] Suppose a path $\beta$ realizes the infimum above. Then $\beta$ is a $\left(\frac{|w|}{|w|-W}, \frac{2 W|w|}{|w|-W}\right)$-quasi-geodesic.

Replace $g_{1}, g_{2}$ by large positive powers if necessary, let $F$ be the subgroup of $G$ generated by $g_{1}, g_{2}$, and let $\Phi: T \rightarrow X$ be an $F$-equivariant map with $\Phi(1)=x_{0}$ as in the proof of Proposition 2. If $w \in F$ is cyclically reduced as a word in $g_{1}, g_{2}$ (equivalently, if its axis passes through $1 \in T$ ) then by the quasi-convexity of $F$ in $G$ we have (see Figure 3 )

$$
d\left(x_{0}, w^{n}\left(x_{0}\right)\right) \geq n\left(d\left(x_{0}, w\left(x_{0}\right)\right)-2 B\right)
$$

where $B=B(K, L, \delta)>0$ is independent of $w$ and $n$.

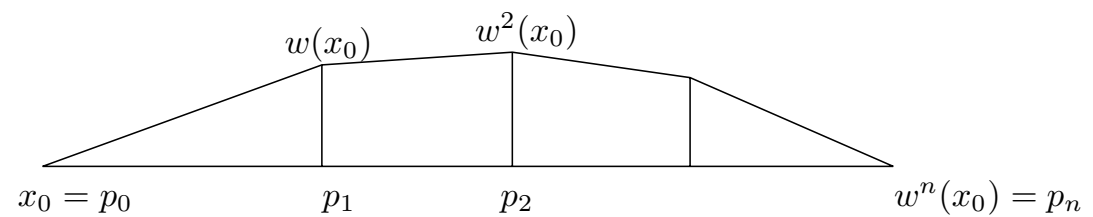

Figure 3: $n d\left(x_{0}, w\left(x_{0}\right)\right) \leq d\left(x_{0}, w^{n}\left(x_{0}\right)\right)+d\left(p_{1}, w\left(x_{0}\right)\right)+d\left(p_{2}, w^{2}\left(x_{0}\right)\right)+\cdots \leq$ $d\left(x_{0}, w^{n}\left(x_{0}\right)\right)+2 n B$

Following [11] we fix an integer $W \geq 3 B$ and will only consider $w$ with $|w|>W$. Thus, paths $\beta$ as in Lemma 3 will be quasi-geodesics with constants independent of $w$ and the endpoints, and $\beta$ is contained in a uniform neighborhood, say $D-$ neighborhood, of any geodesic joining the endpoints of $\beta$. We will also omit $W$ from notation and write $c_{w}$. For every $f \in F$ choose a geodesic $\gamma_{f}$ from $x_{0}$ to $f\left(x_{0}\right)$. We find it convenient to denote the concatenation

$$
\gamma_{f} f\left(\gamma_{f}\right) f^{2}\left(\gamma_{f}\right) \cdots f^{a-1}\left(\gamma_{f}\right)
$$

by $f^{a}$.

Define $h_{w}: G \rightarrow \mathbb{R}$ by

$$
h_{w}(g)=c_{w}\left(x_{0}, g\left(x_{0}\right)\right)-c_{w^{-1}}\left(x_{0}, g\left(x_{0}\right)\right) .
$$


Proposition 4 [11, Proposition 3.10] The map $h_{w}: G \rightarrow \mathbb{R}$ is a quasihomomorphism. Moreover, the defect $\Delta\left(h_{w}\right)$ is uniformly bounded independently of $w$.

Proposition 5 Suppose $1 \neq f \in F$ is cyclically reduced and $f \not f^{-1}$. Then there is $a>0$ such that $h_{f^{a}}$ is unbounded on $\left\langle f>\right.$. Moreover, if $f^{ \pm 1} \not f^{\prime} \in F$ then $h_{f}$ is 0 on $\left\langle f^{\prime}>\right.$ for sufficiently large $a>0$.

Proof It is clear that $c_{f^{a}}$ is unbounded on $\langle f\rangle$ for any $a>0$ : If we use $\alpha=\left(f^{a}\right)^{n}$ as a competitor path we have

$$
c_{f^{a}}\left(f^{a n}\right) \geq d\left(x, f^{a n}(x)\right)-\left(n\left|f^{a}\right|-3 B n\right) \geq B n .
$$

If $a>0$ is large, then there are no copies of $f^{-a}$ in the $D$-neighborhood of an axis of $f$, which implies that $c_{f^{-a}}$ is zero on $\langle f\rangle$.

The proof of the other claim is similar.

Proof of Theorem 1 Let $f_{1}, f_{2}, \cdots$ be the sequence from Proposition 2 . We assume in addition (without loss of generality) that each $f_{i}$ is cyclically reduced. Define $h_{i}: G \rightarrow \mathbb{R}$ as $h_{i}=h_{f^{a_{i}}}$ where $a_{i}>0$ is chosen as in Proposition 5 so that $h_{i}$ is unbounded on $\left\langle f_{i}>\right.$ and so that it is 0 on $<f_{j}>$ for $j<i$ (a high power of $f_{i}$ cannot be translated into a $B$-neighborhood of an axis of $\left.f_{j}\right)$. It follows that $\left[h_{i}\right] \in Q H(G)$ is not a linear combination of $\left[h_{1}\right], \cdots,\left[h_{i-1}\right]$, ie, the sequence $\left[h_{i}\right]$ consists of linearly independent elements. We can easily arrange that $F$ is contained in the commutator subgroup of $G$ (this is automatic if $g_{1}, g_{2}$ are in the commutator subgroup; otherwise, replace $g_{1}, g_{2}$ by $g_{1}^{\prime}=g_{1}^{N} g_{2}^{M} g_{1}^{-M} g_{2}^{-N}, g_{2}^{\prime}=g_{1}^{K} g_{2}^{L} g_{1}^{-K} g_{2}^{-L}$ with $0 \ll N \ll M \ll K \ll L$ - as in Proposition 2 it follows that $\left.g_{1}^{\prime} \nsim g_{2}^{\prime}\right)$. In that case any homomorphism $G \rightarrow \mathbb{R}$ vanishes on $F$ and it follows that the sequence $\left[h_{i}\right]$ in $\widetilde{Q H}(G)$ consists of linearly independent elements.

Remark The argument shows that there is an embedding of $\ell^{1}$ into $Q H(G)$. If $t=\left(t_{1}, t_{2}, \cdots\right) \in \ell^{1}$ then

$$
h_{t}=\sum t_{i} h_{i}: G \rightarrow \mathbb{R}
$$

is a well-defined function $\left(\left|f_{i}\right| \rightarrow \infty\right.$ implies that for any $g \in G$ only finitely many $h_{i}(g)$ are nonzero) and it is a quasi-homomorphism (because the defect of $h_{i}$ is uniformly bounded independently of $i$ ), and $t \neq 0$ implies $h_{t}$ is unbounded (on $\left\langle f_{i}\right\rangle$ where $i$ is the smallest index with $t_{i} \neq 0$ ). Similarly, one can argue that $\widetilde{Q H}(G)$ contains $\ell^{1}$. 
Remark Instead over $\mathbb{R}$ one can work over $\mathbb{Z}$ and consider $H_{b}^{2}(G ; \mathbb{Z})$ and quasi-homomorphisms $G \rightarrow \mathbb{Z}$. The quasi-homomorphisms $h_{w}$ constructed above are integer-valued, and therefore it follows that there are infinitely many linearly independent elements in the kernel of $H_{b}^{2}(G ; \mathbb{R}) \rightarrow H^{2}(G ; \mathbb{R})$ which are in the image of $H_{b}^{2}(G ; \mathbb{Z})$.

\section{$3 \quad$ Weak Proper Discontinuity}

Definition We say that the action of $G$ on $X$ satisfies $W P D$ if

- $G$ is not virtually cyclic,

- $G$ contains at least one element that acts on $X$ as a hyperbolic isometry, and

- for every hyperbolic element $g \in G$, every $x \in X$, and every $C>0$ there exists $N>0$ such that the set

$$
\left\{\gamma \in G \mid d(x, \gamma(x)) \leq C, d\left(g^{N}(x), \gamma g^{N}(x)\right) \leq C\right\}
$$

is finite.

Proposition 6 Suppose that $G$ and $X$ satisfy WPD. Then

(1) for every hyperbolic $g \in G$ the centralizer $C(g)$ is virtually cyclic,

(2) for every hyperbolic $g \in G$ and every $(K, L)$-quasi-axis $\ell$ for $g$ there is a constant $M=M(g, K, L)$ such that if two translates $\ell_{1}, \ell_{2}$ of $\ell$ contain (oriented) segments of length $>M$ that are oriented $B(K, L, \delta)$-close then $\ell_{1}$ and $\ell_{2}$ are oriented $B(K, L, \delta)$-close and moreover the corresponding conjugates $g_{1}, g_{2}$ of $g$ have positive powers which are equal,

(3) the action of $G$ on $X$ is nonelementary,

(4) $f_{1} \sim f_{2}$ if and only if some positive powers of $f_{1}$ and $f_{2}$ are conjugate,

(5) there exist hyperbolic $g_{1}, g_{2}$ such that $g_{1} \nsim g_{2}$.

Proof (1) We will show that $\langle g\rangle$ has finite index in $C(g)$. Let $f_{1}, f_{2}, \cdots$ be an infinite sequence of elements of $C(g)$. Choose a $(K, L)$-quasi-axis $\ell$ for $g$ and let $x \in \ell$. Since $g$ and $f_{i}$ commute, $f_{i}(\ell)$ is also a $(K, L)$-quasi-axis for $g$ and the distance between $\ell$ and $f_{i}(\ell)$ is uniformly bounded by $B(K, L, \delta)$. Let $k_{i} \in \mathbb{Z}$ be such that $d\left(f_{i}(x), g^{k_{i}}(x)\right)$ minimizes the distance between $f_{i}(x)$ and the $g$-orbit of $x$. Thus $d\left(f_{i}(x), g^{k_{i}}(x)\right)$ is uniformly bounded (by $B(K, L, \delta)$ plus the diameter of the fundamental domain for the action of $g$ on $\ell$ ); call such 
a bound $C$. Let $N$ be from the definition of $W P D$. We note that $f_{i} g^{-k_{i}}$ move both $x$ and $g^{N}(x)$ by $\leq C$. Therefore, the set of such elements is finite. From $f_{i} g^{-k_{i}}=f_{j} g^{-k_{j}}$ we conclude that $f_{i}$ and $f_{j}$ represent the same $<g>-$ coset and the claim is proved.

(2) Denote by $g_{1}$ and $g_{2}$ the corresponding conjugates of $g$. For notational simplicity, we will first assume that $g$ is axial and that $\ell$ is an axis of $g$.

Without loss of generality we assume $g_{1}=g$. Choose $x \in \ell$ and let $N$ be as in the definition of $W P D$ for $g, x, C=4 \delta$. Let $P$ be the size of the finite set from the definition of $W P D$. If $\ell_{1}=\ell$ and $\ell_{2}$ contain oriented $2 \delta$-close $\operatorname{arcs} J_{1}$ and $J_{2}$ of length $>(P+N+2) \tau_{g}\left(\tau_{g}\right.$ is the translation length of $\left.g\right)$ then the elements $g_{1}^{i} g_{2}^{-i}$ move each point of the terminal subarc of $J_{2}$ of length $(N+2) \tau_{g}$ a distance $\leq 4 \delta$ for $i=0,1, \cdots, P$. It follows that $g_{1}^{i} g_{2}^{-i}=g_{1}^{j} g_{2}^{-j}$ for distinct $i, j$ so that $g_{1}, g_{2}$ have equal positive powers.

In general, when $\ell$ is only a quasi-axis, one can generalize the above paragraph by replacing $4 \delta$ etc. by larger constants that depend on $(K, L)$ and $\delta$. Alternatively, one can modify $X$ to make $g$ axial: simply attach an infinite ladder (the 1-skeleton of an infinite strip) along one of the two infinite lines to $\ell$; then attach such ladders equivariantly to obtain a $G$-space. Finally, subdivide each rung and each edge in $X$ into a large number $Q$ of edges in order to arrange that the "free" lines in the attached ladders are geodesics and axes for the corresponding conjugates of $g$. The group $G$ continues to act on the new space $X^{\prime}$ which is quasi-isometric to $X$. The statement for $X^{\prime}$ implies the statement for $X$.

(3) Let $g$ be a hyperbolic element. Again, without loss of generality, we will assume that $g$ has an axis $\ell$. We aim to show that some translate of $\ell$, say $h(\ell)$, has both ends distinct from $\ell$, since then $g$ and $h g h^{-1}$ are independent hyperbolic elements.

Suppose first that there is $h \in G$ such that $h(\ell)$ is not in the $2 \delta$-neighborhood of $\ell$, but it is asymptotic in one direction, ie, a ray in $h(\ell)$ is contained in the $2 \delta$-neighborhood of $\ell$. From (2) we see that $\ell$ and $h(\ell)$ cannot contain segments of length $>M(g)$ that are oriented $2 \delta$-close to each other; in particular, one of $g, h g h^{-1}$ moves towards the common end, say $\infty$, and the other moves away from it. Now consider $g^{N}(h(\ell))$ for large $N$. This is a bi-infinite geodesic with one end $\infty$ and the other end distinct from the ends of $\ell, h(\ell)$. The translates $h(\ell)$ and $g^{N}(h(\ell))$ violate $(2)$ as they have oriented rays within $2 \delta$ of each other.

It remains to consider the case when every translate of $\ell$ is within $2 \delta$ of $\ell$. After passing to a subgroup of $G$ of index 2 if necessary, we may assume that 
$G$ preserves the ends of $\ell$. Now proceed as in (1) to show that $\langle g\rangle$ has finite index in $G$.

(4) This is similar to (2). We assume for simplicity that $f_{1}, f_{2}$ are axial. By $\tau_{k}$ denote the translation length of $f_{k}, k=1,2$. Let $N$ be as in the definition of $W P D$ for $g=f_{1}$ with respect to some $x$ in an axis $\ell$ of $f_{1}$ and $C=4 \delta$. Let $P$ be the size of the corresponding finite set. Now assume that $f_{2}$ has an axis that admits a segment of length $>(N+2) \tau_{1}+P \tau_{1} \tau_{2}$ which is oriented $2 \delta$-close to a segment in $\ell$. Consider $f_{2}^{i \tau_{1}} f_{1}^{-i \tau_{2}}, i=0,1, \cdots, P$. As before, we conclude that $f_{2}^{i \tau_{1}} f_{1}^{-i \tau_{2}}=f_{2}^{j \tau_{1}} f_{1}^{-j \tau_{2}}$ for some $i \neq j$; thus $f_{1}$ and $f_{2}$ have common positive powers.

(5) Since the action of $G$ on $X$ is nonelementary, we can choose a Schottky subgroup $F \subset G$. Let $1 \neq f \in F$. For notational simplicity we will assume that all nontrivial elements of $F$ are axial and in fact that there is an $F$-invariant totally geodesic tree $T \subset X$ (this can be arranged by modifying $X$ as in the proof of (2) except that now one attaches the 1 -skeleton of (tree) $\times I$ instead of (line) $\times I)$. Let $\ell \subset T$ be an axis of $f$. Then $W P D$ provides a segment $J$ in $\ell$ such that the set of $g \in G$ that move each point of $J$ by $\leq \tau_{f}+2 \delta$ is finite.

Now consider an infinite sequence $f_{1}, f_{2}, \cdots$ of elements of $F$ with distinct (and hence non-parallel) axes $\ell_{1}, \ell_{2}, \cdots \subset T$ that overlap $\ell$ in (oriented) finite intervals that contain $J$. If $f_{i} \sim f$ then, according to (4), there is $g_{i} \in G$ such that $g_{i}\left(\ell_{i}\right)$ is $2 \delta$-close to $\ell$. Replacing $g_{i}$ by $f^{a_{i}} g_{i}$ if necessary, we may assume that $g_{i}$ moves each point of $J$ by $\leq \tau_{f}+2 \delta$. Thus $g_{i}=g_{j}$ for some $i \neq j$, so that $\ell_{i}$ and $\ell_{j}$ are within $2 \delta$ of each other, contradicting the choice of the sequence.

Finally, note that we could have taken $f_{i}=g f^{i}$ for some $g \in F$ that does not commute with $f$, and that the argument shows that $f_{i} \not f$ for all but finitely many $i$.

Theorem 7 Suppose that $G$ and $X$ satisfy $W P D$. Then $\widetilde{Q H}(G)$ is infinite dimensional.

Proof This is a consequence of Theorem 1 and Proposition 6 .

In order to avoid passage to finite index subgroups, we will need a slight extension of Theorem 7 .

Theorem 8 Suppose that $G$ and $X$ satisfy $W P D$. For $p \geq 1$ form the semidirect product $\tilde{G}=G^{p} \rtimes S_{p}$ where $G^{p}=G \times G \times \cdots \times G$ is the $p$-fold cartesian 
product and $S_{p}$ is the symmetric group on $p$ letters acting on $G^{p}$ by permuting the factors. Let $\tilde{H}<\tilde{G}$ be any subgroup and let $H=\tilde{H} \cap G^{p}$ (subgroup of $\tilde{H}$ of index $\leq p$ !). Thus $H$ has $p$ actions on $X$ obtained by projecting to various coordinates. If at least one of these actions satisfies WPD (equivalently, it is nonelementary) then $\widetilde{Q H}(\tilde{H})$ is infinite dimensional.

Note that Theorem 7 implies that $\widetilde{Q H}(H)$ is infinite dimensional.

Proof The details of this proof are similar to the proof of Theorem 7 and we only give a sketch. We will use the following principle in this proof. If $F$ is a rank 2 free group and $\phi: F \rightarrow G$ a homomorphism then there is a rank 2 free subgroup $F^{\prime}<F$ such that either $\phi\left(F^{\prime}\right)$ contains no hyperbolic elements or else $\phi$ is injective on $F^{\prime}$ and $\phi\left(F^{\prime}\right)$ is Schottky (it follows from WPD that either $\phi(F)$ contains two independent hyperbolic elements, in which case the latter possibility can be arranged, or $\phi(F)$ contains no hyperbolic elements, or $\phi(F)$ is virtually cyclic, and then the first possibility holds).

Say the first projection of $H$ induces an action which is $W P D$. Therefore there is a free group $F=<x, y>\subset H$ such that the first projection of $F$ is Schottky. Now apply the above principle with respect to each coordinate to replace $F$ by a subgroup so that each coordinate action is either Schottky or has no hyperbolic elements. For concreteness, we assume that coordinates $1,2, \cdots, k$ are Schottky and $k+1, \cdots, p$ have no hyperbolic elements $(1 \leq k \leq p)$. We still call $x$ and $y$ the basis elements of $F$.

We will adopt the convention in this proof that for $f \in F$ the $r^{t h}$ projection of $f$ is denoted by $r$.

The proof of Proposition 6(5) (see the last sentence) shows that after replacing $y$ by $x y^{N}$ for some $N$ if necessary, we may assume that ${ }_{r} x{ }_{r} y$ for $r=$ $1,2, \cdots, k$. Next, elements $f=x^{n_{1}} y^{m_{1}} x^{k_{1}} y^{-l_{1}}$ and $g=x^{n_{2}} y^{m_{2}} x^{k_{2}} y^{-l_{2}}$ for $0 \ll n_{1} \ll m_{1} \ll k_{1} \ll l_{1} \ll n_{2} \ll m_{2} \ll k_{2} \ll l_{2}$ will have the property that ${ }_{r} f \not{ }_{r} g^{ \pm 1}$ for $r=1,2, \cdots, k$ (see Claims 1 and 2 in the proof of Proposition 2). We could then construct a sequence $f_{1}, f_{2}, \cdots$ as in the proof of Proposition 2 (in the same manner as in the previous sentence) so that ${ }_{r} f_{i} \chi_{r} f_{j}^{ \pm 1}$ for $i \neq j$ and ${ }_{r} f_{i} \chi_{r} f_{i}^{-1}$.

In addition, we want to arrange that ${ }_{1} f_{j} \chi_{r} f_{j}{ }^{-1}$ for $j=1,2, \cdots$ (note that we cannot hope to arrange ${ }_{1} f_{j} \chi_{r} f_{j}$ since $G$ might have the same $1^{\text {st }}$ and $r^{\text {th }}$ projections). This can be done by modifying the expression for $f_{j}$ so that it reads (for example)

$$
f_{j}=x^{-s_{j}} y^{-t_{j}} x^{n_{j}} y^{m_{j}} x^{k_{j}} y^{-l_{j}}
$$


with $0 \ll s_{1} \ll t_{1} \ll n_{1} \ll m_{1} \ll k_{1} \ll l_{1} \ll s_{2} \ll \ldots$. The idea is that ${ }_{1} f_{j} \sim{ }_{r} f_{j}{ }^{-1}$ would force the situation where a long string of ${ }_{1} y$ 's is close to both a long string of ${ }_{r} x$ 's and a long string of ${ }_{r} x^{-1}$ 's, implying ${ }_{r} x \sim{ }_{r} x^{-1}$. Of course, it can be arranged that this is false by replacing $(x, y)$ with $\left(f_{1}, f_{2}\right)$ from the previous paragraph.

We now define quasi-homomorphisms $h_{i}: G^{p} \rightarrow \mathbb{R}$ by the formula

$$
h_{i}\left(g_{1}, g_{2}, \cdots, g_{p}\right)=h_{\left(1 f_{i} a_{i}\right)}\left(g_{1}\right)+\cdots+h_{\left({ }_{1} f_{i} a_{i}\right)}\left(g_{p}\right)
$$

for large $a_{i}$. These maps clearly extend to quasi-homomorphisms on $\tilde{G}=$ $G^{p} \rtimes S_{p}$. The first summand in the above formula is unbounded on the cyclic subgroup $\left\langle f_{i}\right\rangle$ and it is positive on large positive powers of $f_{i}$. The second through $k^{\text {th }}$ summands are nonnegative on large powers of $f_{i}$ thanks to the fact that ${ }_{1} f_{i} \chi_{r} f_{i}^{-1}$ for $k \geq r>1$. Finally, the other summands are bounded on $\left\langle f_{i}\right\rangle$ since ${ }_{r} f_{i}$ is not hyperbolic for $r>k$. Thus $h_{i}$ is unbounded on $\left\langle f_{i}\right\rangle$. A similar argument shows that $h_{i}$ is bounded on $\left\langle f_{j}>\right.$ for $j<i$, so that the elements of $Q H(\tilde{H})$ induced by $h_{1}, h_{2}, \cdots$ are linearly independent. By choosing the $f_{i}$ 's to lie in the commutator subgroup of $G$ as before, we obtain an infinite linearly independent set in $\widetilde{Q H}(F)$ and hence in $\widetilde{Q H}(\tilde{H})$.

\section{Mapping class groups}

Let $S$ be a compact orientable surface of genus $g$ and $p$ punctures. We consider the associated mapping class group $M C G(S)$ of $S$. This group acts on the curve complex $X$ of $S$ defined by Harvey [17] and successfully used in the study of mapping class groups by Harer [16], [15] and by N V Ivanov [18], [19]. For our purposes, we will restrict to the 1-skeleton of (the barycentric subdivision of) Harvey's complex, so that $X$ is a graph whose vertices are isotopy classes of essential, nonparallel, nonperipheral, pairwise disjoint simple closed curves in $S$ (also called curve systems) and two distinct vertices are joined by an edge if the corresponding curve systems can be realized simultaneously by pairwise disjoint curves. In certain sporadic cases $X$ as defined above is 0 -dimensional or empty (this happens when there are no curve systems consisting of two curves, ie, when $g=0, p \leq 4$ and when $g=1, p \leq 1)$. In the theorem below these cases are excluded (one could rectify the situation by declaring that in those cases two vertices are joined by an edge if the corresponding curves can be realized with only one intersection point). The mapping class group $M C G(S)$ acts on $X$ by $f \cdot a=f(a)$.

$\mathrm{H}$ Masur and Y Minsky proved the following remarkable result. 
Theorem 9 [22] The curve complex $X$ is $\delta$-hyperbolic. An element of $M C G(S)$ acts hyperbolically on $X$ if and only if it is pseudo-Anosov .

The following lemma is well-known (see [6, Theorem 2.7]).

Lemma 10 Suppose that $a$ and $b$ are two curve systems on $S$ that intersect minimally and such that $a \cup b$ fills $S$. Then the intersection $S(a, b)$ of the stabilizers of $a$ and of $b$ in $M C G(S)$ is finite.

We remark that $a \cup b$ fills $S$ if and only if $d(a, b) \geq 3$ in the curve complex.

Proof Let $g$ be in the stabilizer of both $a$ and $b$. Then there is an isotopy of $g$ so that $g(a)=a$ and $g(b)=b$. It follows that for some $N>0$ depending only on the complexity of the graph $a \cup b$ we have that $g^{N}$ is isotopic to the identity. Therefore $S(a, b)$ consists of elements of finite order and is consequently finite (every torsion subgroup of a finitely generated virtually torsion-free group is finite).

Proposition 11 Let $S$ be a nonsporadic surface. The action of $M C G(S)$ on the curve complex $X$ satisfies $W P D$.

Proof The first two bullets in the definition of $W P D$ are clear. Our proof of the remaining property is motivated by Feng Luo's proof (as explained in [22]) that the curve complex has infinite diameter. We recall the construction and the basic properties of Thurston's space of projective measured foliations on $S$ (see [26] and [10]). Let $\mathcal{C}$ be the set of all curve systems in $S$ and by

$$
I: \mathcal{C} \times \mathcal{C} \rightarrow[0, \infty)
$$

denote the intersection pairing, ie, $I(a, b)$ is the smallest number of intersection points between $a$ and $b$ after a possible isotopy. Let $\mathbb{R}_{+}=(0, \infty)$ and by $\mathbb{R}_{+} \mathcal{C}$ denote the space of formal products $t a$ for $t \in \mathbb{R}_{+}$and $a \in \mathcal{C}$ where we identify $\mathcal{C}$ with the subset $1 \mathcal{C}$. Extend $I$ to $\mathbb{R}_{+} \mathcal{C} \times \mathbb{R}_{+} \mathcal{C}$ by

$$
I(t a, s b)=t s I(a, b) .
$$

Consider the associated function

$$
J: \mathbb{R}_{+} \mathcal{C} \rightarrow[0, \infty)^{\mathcal{C}}
$$

defined by

$$
J(t a)=(s b \mapsto I(t a, s b)) .
$$


Then $J$ is injective and we let $\mathcal{M F}$ denote the closure of the image of $J$. An element of $\mathcal{M F}$ can be viewed as a measured foliation on $S$. The pairing $I$ extends to a continuous function

$$
I: \mathcal{M F} \times \mathcal{M F} \rightarrow[0, \infty) .
$$

There is a natural action of $\mathbb{R}_{+}$on $\mathcal{M F}$ given by scaling. The orbit space $\mathcal{P} \mathcal{M F}$ is Thurston's space of projective measured foliations and it is homeomorphic to the sphere of dimension $6 g+2 p-7$ (assuming this number is positive). The intersection pairing is not defined on $\mathcal{P} \mathcal{M F} \times \mathcal{P} \mathcal{M F}$ but note that the statement $I\left(\Lambda, \Lambda^{\prime}\right)=0$ makes sense for $\Lambda, \Lambda^{\prime} \in \mathcal{P} \mathcal{M F}$. The mapping class group $M C G(S)$ of $S$ acts on $\mathcal{C}$ by $f \cdot a=f(a)$ and there is an induced action on $\mathbb{R}_{+} \mathcal{C}, \mathcal{M F}$, and $\mathcal{P} \mathcal{M F}$.

Let $f \in M C G(S)$ be a pseudo-Anosov mapping class. Then $f$ fixes exactly two points in $\mathcal{P} \mathcal{M F}$ and one point $\Lambda_{+}$is attracting while the other $\Lambda_{-}$is repelling. All other points converge to $\Lambda_{+}$under forward iteration and to $\Lambda_{-}$ under backward iteration. It is known that $I\left(\Lambda_{+}, \Lambda\right)=0$ implies $\Lambda=\Lambda_{+}$and similarly for $\Lambda_{-}$. Continuity of $I$ implies the following fact:

If $U$ is a neighborhood of $\Lambda_{+}$then there is a neighborhood $V$ of $\Lambda_{+}$such that if $\Lambda, \Lambda^{\prime} \in \mathcal{P} \mathcal{M F}, I\left(\Lambda, \Lambda^{\prime}\right)=0$ and $\Lambda \in V$ then $\Lambda^{\prime} \in U$.

We will use the terminology that $V$ is adequate for $U$ if the above sentence holds. A similar fact (and terminology) holds for neighborhoods of $\Lambda_{-}$.

Given $C>0$, choose closed neighborhoods $U_{0} \supset U_{1} \supset U_{2} \supset \cdots \supset U_{N}$ of $\Lambda_{+}$ and $V_{0} \supset V_{1} \supset V_{2} \supset \cdots \supset V_{N}$ of $\Lambda_{-}$with $N>C$ so that

- $U_{i+1}$ is adequate for $U_{i}$ and $V_{i+1}$ is adequate for $V_{i}$, and

- if $\Lambda \in U_{0}$ and $\Lambda^{\prime} \in V_{0}$ then $I\left(\Lambda, \Lambda^{\prime}\right) \neq 0$.

Assume now that two curve systems $a$ and $b$ belong to a quasi-axis $\ell$ of a pseudo-Anosov mapping class $f$ and that they are sufficiently far away from each other, so that after applying a power of $f$ and possibly interchanging $a$ and $b$ we may assume that $a \in U_{N}$ and $b \in V_{N}$. Assume, by way of contradiction, that $g_{n}$ is an infinite sequence in $M C G(S)$ and $d\left(a, g_{n}(a)\right) \leq N$, $d\left(b, g_{n}(b)\right) \leq N$ for all $n$. Note that if $c$ is a curve system disjoint from $a$ then $c \in U_{N-1}$, and inductively if $d(a, c) \leq N$ then $c \in U_{0}$. We therefore conclude that $g_{n}(a) \in U_{0}$ and $g_{n}(b) \in V_{0}$. After passing to a subsequence, we may assume that the sequence $g_{n}(a)$ converges to $A \in U_{0}$ and $g_{n}(b) \rightarrow B \in V_{0}$. Note that $I(A, B) \neq 0$ by the choice of $U_{0}$ and $V_{0}$.

First suppose that the curve systems $g_{n}(a)$ are all different. To obtain convergence in $\mathcal{M F}$ one is required to first rescale by some $r_{n}>0$, ie, $\frac{1}{r_{n}} g_{n}(a) \rightarrow \tilde{A} \in$ 
$\mathcal{M F}$ where $r_{n}$ can be taken to be the length of $g_{n}(a)$ in some fixed hyperbolic structure on $S$. Under the assumption that $g_{n}(a)$ are all distinct, we see that $r_{n} \rightarrow \infty$ and this implies that $I(\tilde{A}, \tilde{B})=0$ ie, that $I(A, B)=0$, contradiction. The case when $g_{n}(b)$ are all distinct is similar.

Finally, if $g_{n}(a)$ and $g_{n}(b)$ take only finitely many values, we may assume by passing to a subsequence that both $g_{n}(a)$ and $g_{n}(b)$ are constant. But then $g_{n}^{-1} g_{m} \in S(a, b)$ and Lemma 10 implies that the sequence $g_{n}$ is finite.

The following is the main theorem in this note. $\mathrm{H}$ Endo and D Kotschick [7] have shown using 4-manifold topology and Seiberg-Witten invariants that $\widehat{Q H}(M C G(S)) \neq 0$ when $S$ is hyperbolic. M Korkmaz [21] also proved $\widetilde{Q H}(M C G(S)) \neq 0$, and in addition that $\widetilde{Q H}(M C G(S))$ is infinite dimensional when $S$ has low genus. The nontriviality, and even infinite-dimensionality, of $\widetilde{Q H}(M C G(S))$ was conjectured by Morita [25, Conjecture 6.19].

Theorem 12 Let $G$ be a subgroup of $M C G(S)$ which is not virtually abelian. Then $\operatorname{dim} \widetilde{Q H}(G)=\infty$.

Proof We first deal with the sporadic cases. When $g=0, p \leq 3$ and when $g=1, p=0$ the mapping class group $M C G(S)$ is finite. When $g=0, p=4$ and when $g=p=1$ then $M C G(S)$ is word hyperbolic (in fact, the quotient by the finite center is virtually free) and instead of considering the action on a curve complex we can look at the action on the Cayley graph. This action is properly discontinuous and therefore the restriction to any subgroup which is not virtually cyclic satisfies $W P D$. The statement then follows from Theorem 7 .

Now we assume that $S$ is not sporadic. By the classification of subgroups (see [23, Theorem 4.6]) there are 4 cases.

- $G$ contains two independent pseudo-Anosov homeomorphisms. Then the action of $G$ on the curve complex $X$ for $S$ satisfies the assumptions of Theorem 7 so $\widetilde{Q H}(G)$ is infinite dimensional.

- $G$ fixes a pair $\Lambda_{ \pm}$of foliations corresponding to a pseudo-Anosov homeomorphism. Then $G$ is virtually cyclic.

- $G$ is finite.

- There is a curve system $c$ on $S$ invariant under $G$. Choose $c$ to be maximal possible and cut $S$ open along $c$. Consider the mapping class group of the cut open surface $S^{\prime}$ where we collapse each boundary component 
to a puncture. Since $c$ is maximal and $G$ is not virtually abelian, there is an orbit $S_{1}^{\prime}, S_{2}^{\prime}, \cdots, S_{p}^{\prime}$ of components of $S^{\prime}$ and there is a subgroup of $G$ that preserves these components and whose restriction to a component contains two independent pseudo-Anosov homeomorphisms. Pass to the quotient of $G$ corresponding to the restriction to $S_{1}^{\prime} \cup \cdots \cup S_{p}^{\prime}$. The mapping class group of $S_{1}^{\prime} \cup \cdots \cup S_{p}^{\prime}$ can be identified with $M C G\left(S_{1}^{\prime}\right)^{p} \rtimes S_{p}$ so the result in this case follows from Theorem 8 .

The following is a version of superrigidity for mapping class groups. It was conjectured by N V Ivanov and proved by Kaimanovich and Masur [20] in the case when the image group contains independent pseudo-Anosov homeomorphisms and it was extended to the general case by Farb and Masur [9] using the classification of subgroups of $M C G(S)$ as above. Our proof is different in that it does not use random walks on mapping class groups, but instead uses the work of M Burger and N Monod [5] on bounded cohomology of lattices. Note also that for this application we only need a weak version of our result, namely that $\widetilde{Q H}(G) \neq 0$ when $G \subset M C G(S)$ is not virtually abelian.

Corollary 13 Let $\Gamma$ be an irreducible lattice in a connected semi-simple Lie group $G$ with no compact factors, with finite center, and of rank $>1$. Then every homomorphism $\Gamma \rightarrow M C G(S)$ has finite image.

Proof Let $\phi: \Gamma \rightarrow M C G(S)$ be a homomorphism. By the Margulis-Kazhdan theorem [27, Theorem 8.1.2] either the image of $\phi$ is finite or the kernel of $\phi$ is contained in the center. When $\Gamma$ is a nonuniform lattice, the proof is easier and was known to Ivanov before the work of Kaimanovich-Masur (see Ivanov's comments to Problem 2.15 on Kirby's list). Since the rank is $\geq 2$ the lattice $\Gamma$ then contains a solvable subgroup $N$ which does not become abelian after quotienting out a finite normal subgroup. If the kernel is finite, then $\phi(N)$ is a solvable subgroup of $M C G(S)$ which is not virtually abelian, contradicting [1].

Now assume that $\Gamma$ is a uniform lattice. If the kernel $\operatorname{Ker}(\phi)$ is finite then there is an unbounded quasi-homomorphism $q: \operatorname{Im}(\phi) \rightarrow \mathbb{R}$ by Theorem 12 . But then $q \phi: \Gamma \rightarrow \mathbb{R}$ is an unbounded quasi-homomorphism contradicting the Burger-Monod result that every quasi-homomorphism $\Gamma \rightarrow \mathbb{R}$ is bounded.

Remark When the center $Z(G)$ of $G$ is infinite, one can show that every homomorphism $\phi: \Gamma \rightarrow M C G(S)$ has virtually abelian image, as follows. The key is that in this case the intersection $\Gamma \cap Z(G)$ has finite index in $Z(G)$ and the projection of $\Gamma$ in $G / Z(G)$ is a lattice $L$ in $G / Z(G)$, which is a Lie group of 
rank $>1$. Choose $g \in \Gamma \cap Z(G)$ of infinite order such that the Nielsen-Thurston representative of $\phi(g)$ has no rotations (ie, the canonical invariant curve system cuts the surface into invariant subsurfaces and on each subsurface (with boundary components collapsed to punctures) $\phi(g)$ is isotopic to a pseudo-Anosov homeomorphism or to the identity). There is an induced map $\phi: \Gamma \rightarrow C(\phi(g))$, the centralizer of $\phi(g)$. Moreover, from the Nielsen-Thurston theory we have a homomorphism $\psi: C(\phi(g)) \rightarrow M C G\left(S_{1}\right) \times M C G\left(S_{2}\right) \times \cdots \times M C G\left(S_{k}\right)$ given by restricting to the subsurfaces on which $\phi(g)$ is identity. The kernel of $\psi$ is virtually abelian. So it suffices to argue that the image of the composition $\psi \phi: \Gamma /<g>\rightarrow M C G\left(S_{1}\right) \times M C G\left(S_{2}\right) \times \cdots \times M C G\left(S_{k}\right)$ is virtually abelian. Now $\Gamma /\langle g\rangle$ is a lattice in $G /\langle g\rangle$, a Lie group of rank $>1$ and the rank of the center $Z(G /<g>)=Z(G) /<g>$ is smaller than the rank of $Z(G)$. So the statement follows by induction on the rank of the center.

Remark It can also be shown that the image of $\Gamma \rightarrow M C G(S)$ must be finite, even if $Z(G)$ has infinite center. If the image is infinite, we can assume (by passing to a subgroup of $\Gamma$ of finite index) that it is torsion-free and abelian of finite rank. If $G$ and $\Gamma$ satisfy Kazhdan's property ( $\mathrm{T}$ ), then the abelianization of $\Gamma$ is finite and this is the case when $G$ has no rank 1 factors. It is also true that the abelianization of $\Gamma$ is finite when $G$ is any higher rank group and this follows from a deep work of Prasad-Raghunathan and Deligne.

Remark Theorem 12 also implies that S-arithmetic groups and certain groups of automorphisms of trees do not occur as subgroups of mapping class groups. These are the groups $\Gamma$ for which Burger-Monod show $\widetilde{Q H}(\Gamma)=0$ [5], [3], [4].

Acknowledgments The authors would like to thank Yair Minsky and Gopal Prasad for useful discussions. The first author gratefully acknowledges the support by the National Science Foundation. The second author appreciates the hospitality of the mathematics department of the University of Utah.

\section{References}

[1] J S Birman, A Lubotzky, J McCarthy, Abelian and solvable subgroups of the mapping class group, Duke Math. J. 50 (1983) 1107-1120

[2] Robert Brooks, Some remarks on bounded cohomology, from: "Riemann surfaces and related topics: Proceedings of the 1978 Stony Brook Conference (State Univ. New York, Stony Brook, NY, 1978)", Princeton Univ. Press, Princeton, NJ (1981) 53-63 
[3] M Burger, N Monod, Continuous bounded cohomology and applications to rigidity theory, preprint (2000)

[4] M Burger, N Monod, Mauntner property, Hochschild-Serre sequence in bounded cohomology, and applications, preprint (2000)

[5] M Burger, N Monod, Bounded cohomology of lattices in higher rank Lie groups, J. Eur. Math. Soc. (JEMS) 1 (1999) 199-235

[6] Andrew J Casson, Steven A Bleiler, Automorphisms of surfaces after Nielsen and Thurston, Cambridge University Press, Cambridge (1988)

[7] H Endo, D Kotschick, Bounded cohomology and non-uniform perfection of mapping class groups, Invent. Math. 144 (2001) 169-175

[8] David B A Epstein, Koji Fujiwara, The second bounded cohomology of wordhyperbolic groups, Topology 36 (1997) 1275-1289

[9] Benson Farb, Howard Masur, Superrigidity and mapping class groups, Topology 37 (1998) 1169-1176

[10] A Fathi, F Laudenbach, V Poenaru, Travaux de Thurston sur les surfaces, Société Mathématique de France, Paris (1979), séminaire Orsay, With an English summary

[11] Koji Fujiwara, The second bounded cohomology of a group acting on a Gromovhyperbolic space, Proc. London Math. Soc. (3) 76 (1998) 70-94

[12] Koji Fujiwara, The second bounded cohomology of an amalgamated free product of groups, Trans. Amer. Math. Soc. 352 (2000) 1113-1129

[13] M Gromov, Hyperbolic groups, from: "Essays in group theory", Springer, New York (1987) 75-263

[14] Michael Gromov, Volume and bounded cohomology, Inst. Hautes Études Sci. Publ. Math. (1982) 5-99 (1983)

[15] John L Harer, Stability of the homology of the mapping class groups of orientable surfaces, Ann. of Math. 121 (1985) 215-249

[16] John L Harer, The virtual cohomological dimension of the mapping class group of an orientable surface, Invent. Math. 84 (1986) 157-176

[17] W J Harvey, Boundary structure of the modular group, from: "Riemann surfaces and related topics: Proceedings of the 1978 Stony Brook Conference (State Univ. New York, Stony Brook, NY, 1978)", Princeton Univ. Press, Princeton, NJ (1981) 245-251

[18] N V Ivanov, Complexes of curves and Teichmüller spaces, Mat. Zametki 49 (1991) 54-61, 158

[19] Nikolai V Ivanov, Automorphism of complexes of curves and of Teichmüller spaces, Internat. Math. Res. Notices (1997) 651-666

[20] Vadim A Kaimanovich, Howard Masur, The Poisson boundary of the mapping class group, Invent. Math. 125 (1996) 221-264 
[21] M Korkmaz, Commutators in mapping class groups and bounded cohomology, preprint (2000) arxiv:math.GT/0012162

[22] Howard A Masur, Yair N Minsky, Geometry of the complex of curves. I. Hyperbolicity, Invent. Math. 138 (1999) 103-149

[23] John McCarthy, Athanase Papadopoulos, Dynamics on Thurston's sphere of projective measured foliations, Comment. Math. Helv. 64 (1989) 133-166

[24] Nicolas Monod, Continuous bounded cohomology of locally compact groups, Springer-Verlag, Berlin (2001)

[25] Shigeyuki Morita, Structure of the mapping class groups of surfaces: a survey and a prospect, from: "Proceedings of the Kirbyfest (Berkeley, CA, 1998)", Geom. Topol. Monogr. 2 (1999) 349-406

[26] William $\mathbf{P}$ Thurston, On the geometry and dynamics of diffeomorphisms of surfaces, Bull. Amer. Math. Soc. 19 (1988) 417-431

[27] Robert J Zimmer, Ergodic theory and semisimple groups, Birkhäuser Verlag, Basel (1984) 